\title{
Study on Extenics Information Fusion Method
}

\author{
Li Zhong $^{\mathrm{a}}$,Wang Ailing ${ }^{\mathrm{b}}$,Qi Furong ${ }^{\mathrm{c}}$ \\ ${ }^{a, b, c}$ Institute of Disaster Prevention Science and Technology. Sanhe, Hebei. China
}

\begin{abstract}
This paper makes analysis on theories of D-S evidential reasoning, rough sets and extenics sets, the three of which hold similarities in defining uncertainty sets. It's feasible to introduce relevant theories and methods of extenics into information fusion and a method of extenics fusion (MEF) is presented as well. The method combines extenic correlation function with Dempster method and is considered to be a good solution in solving evidence collision and BPA function in information fusion method basing on D-S evidential reasoning. With the difference of transfer functions, the paper designs a method of extenics fusion. The simulation test results show that MEF is better than the traditional D-S evidential reasoning and is applicable to all kinds of evaluation problems.
\end{abstract}

Keywords- Extenics; Information fusion; MEF

(C) 2011 Published by MECS Publisher. Selection and/or peer review under responsibility of the International Conference on E-Business System and Education Technology

\section{Introduction}

In information fusion algorithm based on D-S evidential reasoning, it is often required to compute the basic probability distribution functions (BPA), belief function and plansibility function for all basic evidential bodies. The Dempster rule of combination can put the belief functions of each evidential body together, compute the orthogonal sum of confidence levels from different sources, obtain a new belief function on the target body and form a correct judgment on the objective. It has been difficulty how to choose a proper BPA in the information fusion algorithm based on D-S evidential reasoning, however, whether the BPA is accuracy or not brings a direct influence on the fusion result. For this reason, some scholars apply soft methods to obtain BPA and have got some effect [1-3]. Yet, these methods are very complex and not intuitive in calculation. The Dempster rule requires that the evidential body is independent to each other. Often when conflicts occur, it will be possible inconsistency between fusion results and intuition. The conflicting evidential bodies need to be revised [4] and the correlation evidential body is converted into independent ones before using Dempster rule. Yet the BPA functions still need to be computed when obtaining the correlation coefficient. Therefore, it is worth to make further study on how to obtain a reasonable BPA intuitively and quickly as well as to resolve the conflicts of evidential bodies.

The Extenics is better used in solving incompatibilities and conflicts [6]. With matter-element theory and

Corresponding author:

E-mail address: lizhong@fzxy.edu.cn 
correla- tion function, the extenics usually unites qualitative and quantitative changes together to handle conflicts. This paper introduces the extenics theory into information fusion calculations, defines BPA of D-S evidential reasoning with extenics correlation functions, and combines all BPA with Dempster rule, which makes it simple and feasible to determine the BPA of evidential bodies.

\section{COMPARISON OF D-S EVIDENCE THEORY,ROUGH SET THEORY AND EXTENICS SET THEORY}

Some basic concepts adopted in the information fusion based on D-S evidential reasoning mainly includes basic probability assignment function, belief function and plansibility function, among which, the belief function describes the maximum intervals value of supporting evidence and the plansibility function characterizes the minimum intervals value of unsupported evidence, the remaining part between the two values is the believable interval of supporting evidence, $[\operatorname{Bel}(A), P l(A)]$, as shown in Fig. 1. It can be seen that the believable interval has been an uncertain set in the D-S evidential reasoning and the fusion computing is in the belief interval.

In the rough set theory, two identified concepts of upper and lower approximation can be used to describe the fuzzy of uncertainty sets. The difference between the two sets is regarded as the uncertainty set, i.e, the boundary field. According to rough set theory, the lower approximation set is called positive field, the complement set of upper approximation is called negative field, the difference between the two sets is called the boundary field. The relationship of the three field is shown in Fig. 2.

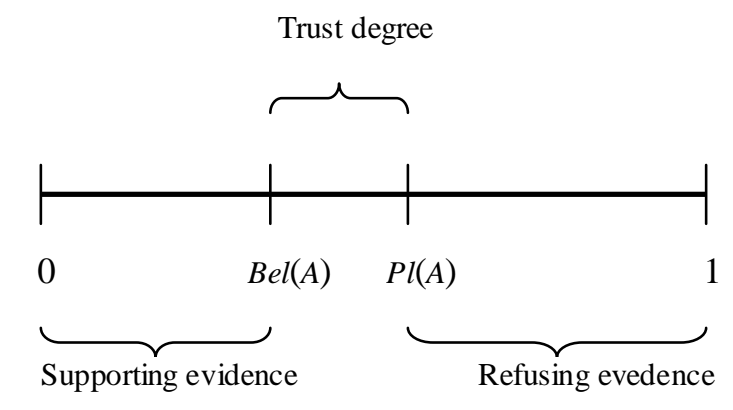

Fig.1 Believable interval of D-S evidence theory

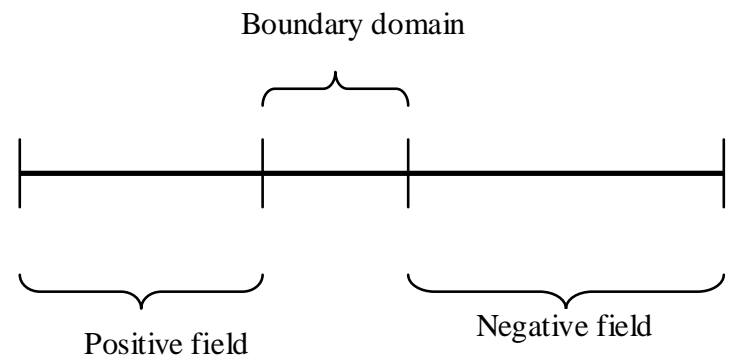

Fig.2 Positive field, negative field and boundary field of rough set theory

Teacher Research Fund of China Seismological Bureau (No.20090105) and Science and Research Programs of Hebei Education Department of China(No. Z2009407) 
Zero boundary

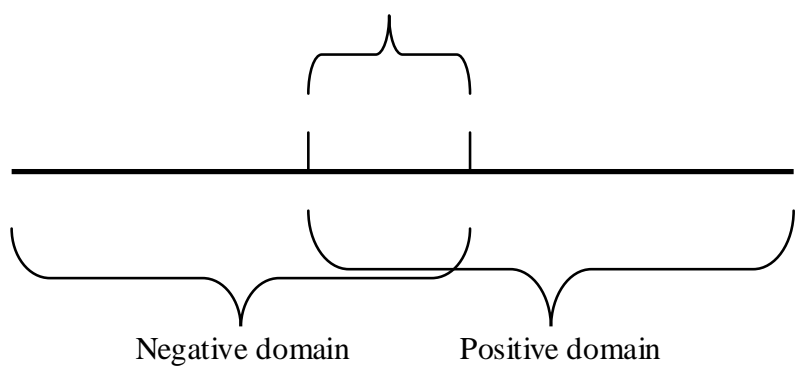

Fig.3 Positive field, negative field and zero field of extenic set theory

The Extenics set can be divided into three parts, positive field, negative field and zero boundary.The positive field represents matter-element set that meets requirements, while the negative field refers to the sets that does not meet requirements. The zero boundary means intersection of positive and negative fields, which refers to a specious concept and the state can be changed to satisfied from unsatisfied after a certain transformations as shown in Fig. 3.

From the above description, it is easily seen that there are lots of similarities for the three kinds of sets in characterizing uncertain sets. The D-S evidence theory uses confidence interval to show uncertainty sets and the rough set theory names the uncertainty interval as a boundary field, while the extenics set theory refers the zero boundary as the uncertainty interval. Since both interval of $(-\infty,+\infty)$ and $(0,1)$ have isomorphism relation, the definition of the three are accordant. As we all know that the D-S evidence theory and rough set theory have been widely used in information fusion, it is feasible to introduce the extenics theory into information fusion processing with some matured research methods.

\section{INFORMATION FUSION METHOD OF EXTENICS-BASED D-S EVIDENTIAL THEORY}

Factors affecting change of things usually cover many aspects and the common information accumulation from every single aspect causes qualitative change of things. So we need to make composite consideration for all information of every channel. Shafer said [7]: "The influence on one proposition of evidence should include at least two aspects of information: how the evidence effectively proves the proposition and how the evidence effectively proves its anti-proposition". In the D-S evidential theory, it is difficult to determine the confidence level of one proposition, and usually possesses subjectivity. The probability of digitalization cannot be independent of the objective attributes of human judgments, which not exist in our brains before thinking. However, we can construct a digitalized probability with basis of objective evidences as well as make analysis over the evidences during the whole process. Perhaps the evidences are ambiguous in the very beginning and the judgment about propositions is also non-quantifiable, even without any structure and logic, yet, with advancement of understanding and appearance of other evidences, we may identify the reliability level for a related digitalized proposition [5]. While in the extenics, the correlation function can easily characterize the correlation degree between evidence and proposition. So we can introduce the correlation function of extenics theory into the D-S evidential theory to indicate the supporting degree of evidence to a proposition through the degree of correlation between the character value and classical domain or joint domain of matter element. 
The analysis shows that a link can be established between extenics correlation function and belief function in D-S evidential theory. So, a simple method can be found to determine the confidence degree when defining the supporting degree of evidence to proposition with their correlation degree to each other, which is also a practical solution in addressing the basic probability assignment in D-S theory. The algorithm of introducing extenics theory to the information fusion is called method of extenics fusion (MEF).

Based on the above analysis, algorithm is designed as follows:

Step1. Setting matter-element model

According to the definition of matter-element, it is limitless to describe the characteristics of a thing, but to describe a thing with only finite characteristics. Supposing that there are $n$ features of a thing, denoted as $\left\{C_{1}\right.$, $\left.C_{2}, \ldots, C_{n}\right\}$. So the element-model is set up as follows:

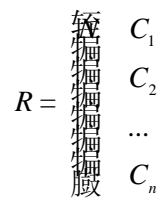

Step2. Determining the classical domain of matter- element

According to the matter-element theory, the scope of typical value for a thing in keeping its own characteristic is called to be classical domain of matter element. The classical domain can be divided into multiple subfields under its different characteristics of matter element. Each subfield is called level or rank for the purpose of detailed analyzing matters. The classical domain of matter-element is generally an interval consisted by a pair of data. For the non-digital text, a digital processing needs to be done to calculate facilitately. Let's suppose the matter-element classical domain with n-feature $\left\{C_{1}, C_{2}, \ldots, C_{n}\right\}$ is as follows:

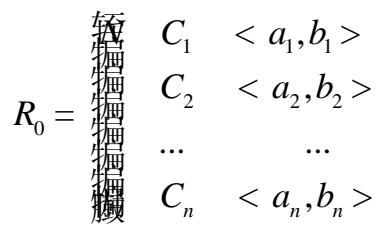

A classification is usually done in evaluating problems. Supposing the target is divided into $\mathrm{m}$ levels with $\left\{\mathrm{I}_{1}\right.$, $\left.\mathrm{I}_{2}, \ldots, \mathrm{I}_{m}\right\}$ of the corresponding classical domain, which can be presented with matrix as follows:

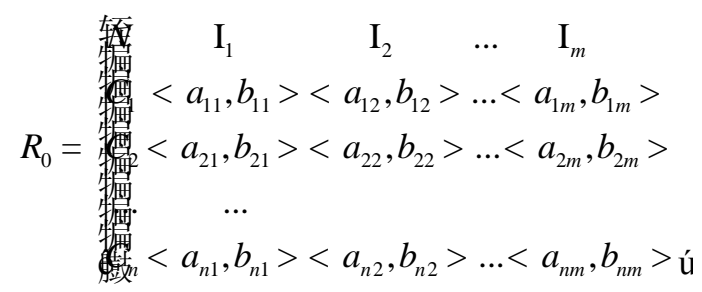

Step3. Determining the joint domain of matter- element

The joint domain of matter-element is the scope of the quantitative change which keeps its quality. When the variation of quantity is within the scope of joint domain, the quality will maintain its stability, once beyond the joint domain, a qualitative change will happen. So, the joint domain is the union of all classical domains. If expressed with above matter-element, then the joint domain will be: 


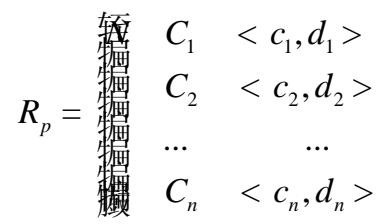

Clearly, according to the level classical domains, there is $c_{i}=a_{i 1}, d_{i}=b_{i m}, i=1,2, \ldots, n$.

Step4. Determining the matter-element to be evaluated

The characteristics of matter-element can be obtained through inspection, measure, test, experience, analysis, computation and other ways. Assuming the matter-element to be evaluated is:

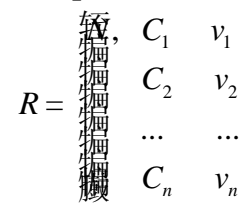

Step5. Computing the correlation degree of matter- element for classical domains

Assuming that the classical domain of feature $\mathrm{C}$ for matter-element $\mathrm{N}$ is $\langle\mathrm{a}, \mathrm{b}\rangle$, the joint domain is $\langle\mathrm{c}, \mathrm{d}\rangle$, the corresponding value to be evaluated is $v$, and so the correlation degree can be computed as follows:

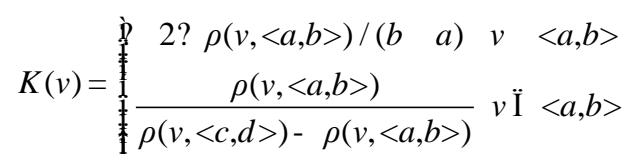

Here, $\rho(v,\langle a, b>)=|v-(a+b) / 2|-(b-a) / 2$

$$
\rho(v,\langle c, d>)=|v-(c+d) / 2|-(d-c) / 2
$$

For evaluation, the above correlation value will form a matrix of $n \times m$ that the column represents feature, while the row means correlation degree. That is:

$$
\begin{aligned}
& \begin{array}{llll}
\mathrm{I}_{1} & \mathrm{I}_{2} & \ldots & \mathrm{I}_{m}
\end{array}
\end{aligned}
$$

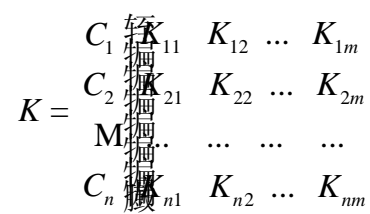

Step6. Calculating the belief degree of evidential body

An evidential body consists $(A, \mathrm{~m}(A))$, where $A$ is proposition and $\mathrm{m}(A)$ is the reliability distribution function supporting $A$. With the correlation function of classical domain for the matter-element, we can obtain the belief degree of a proposition, i.e; one feature value belongs to a specific classical domain. Supposing $k$ is the correlation degree between a feature value $v$ and one classical domain, there is $-? k<+$ from the definition of correlation function of (5). Using S-function to transform and making normalization with row:

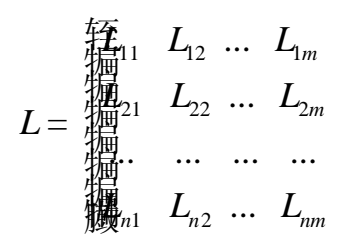

Thus, in (7), the element in each row is the distribution of confident degree for feature value in supporting 
classification proposition.

Step7. Making information fusion using Dempster rule

According to Dempster rule [7], the confident degree of different evidential bodies shall be combined a whole distribution of credibility level, which produces a new evidential body. Thus the final recognition result is generated as well.

\section{EXPERIMENTAL SIMULATION AND ANALYSIS OF MEF ALGORITHM}

Experimental simulation is needed to verify the validity of the extenics fusion as well as an analysis over the data and making comparison with the result of other method.

\section{1 Experimental simulation of MEF algorithm}

To achieve MEF, we developed a simulation program using Matlab and made verification as an example with data provided by literature [8].

Tab.1 shows the surface water indexes of one river during the first three months in 2002. Each test item was a group of evidence in water quality checking. The surface water is divided into five categories according to national standards [9]. Due to the bigger of dissolved oxygen the better among all testing items, we used its countdown for processing data.

Tab.1 Water quality index of a river $(m g / \mathrm{L})$

\begin{tabular}{|c|c|c|c|c|}
\hline & BOD & Permanganate & $\begin{array}{c}\text { Dissolved } \\
\text { xygen }\end{array}$ & Ammonia \\
\hline Jan. & 0.95 & 2.28 & 10.8 & 0.213 \\
\hline Feb. & 1.58 & 2.55 & 10.55 & 0.387 \\
\hline Mar. & 0.9 & 3.03 & 10.5 & 0.37 \\
\hline
\end{tabular}

In the information fusion of D-S evidential theory, we need to know the basic belief level of each sample with regard to the surface water level and make fusion calculation using data of literature [8]. For purpose of comparison, we conducted a water quality evaluation with 3 methods (D-S evidential theory, extenics evaluation and MEF). The results are shown in Tab. 2.

Tab.2 Surface water assessment results for a river from Jan.to Mar.

\begin{tabular}{|l|c|c|c|}
\hline & Jan. & Feb. & Mar. \\
\hline $\begin{array}{l}\text { D-S evidential } \\
\text { theory }\end{array}$ & I & I & I \\
\hline $\begin{array}{l}\text { Extenics } \\
\text { evaluation }\end{array}$ & I & II & II \\
\hline MEF & I & II & II \\
\hline
\end{tabular}

As can be seen in Tab.2, the results of extenics evaluating method and extenics fusion are consistent, while result of D-S evidential theory is different.

The analysis shows $\mathrm{D}-\mathrm{S}$ evidential theory requires a basic reliability function value of all evidence 
bodies as s base, while the values are usually given artificially, which consequentially leads to the inaccuracy of judgment.

\section{2 Algorithm analysis}

Firstly, misjudgment will happen often due to the improper handling to the conflicting evidence of D-S evidence theory. In the multi-source information fusion, since the manifold information delivers diversity and uncertainty of information, this is the root cause of why we use information fusion. However, the diversity and uncer- tainty of information brings more conflicting possibilities to the source evidence. This kind of conflict is not caused by a single focal element, it could be the errors of two different evidences, or some unknown reason or uncertainties, or some external disturbances. According to Dempster rule, there is no processing when evidence conflicting happened, which would produce inconsistent conclusion compared with intuition. In fact, the debate on the Dempster rule has always existed in the field of information fusion and currently there are two main options. One option believes the Dempster rule is improper in dealing with two intersectional BPA functions of focal elements under a condition of empty set and results misjudgment. The solution to this problem is to redistribute the conflicted evidence [4]. The other option believes that the Dempster rule is not wrong. Yet, a preprocessing shall be done to the evidence when conflicts happened in order to prevent conflicts from occurrence [7]. The extenics-based informa- tion fusion method of this paper belongs to the latter option.

The second is robust performance. When small changes occur in giving basic probability assignment value to a focal element, the results of Dempster rule will change sharply and show weak stability, which reflect that the Dempster rule is extremely sensitive to the values of basic belief function.

Lastly, the computing complexity of algorithm. The MEF consists two parts in calculation: confidence distribution of category proposition and Dempster combining calculation. The main work in the first part is to obtain consumption of correlation matrix with a computation of $m n+m n+m n=O(m n)$. In the second part, the computation of information fusion for $n$ sensors is $O\left(n^{2}\right)$, taking into account $m$, the evaluation category and $O\left(m n^{2}\right)$, parts of the computation amount of information fusion. So the total computation is $O(m n)+O\left(m n^{2}\right)=$ $O\left(m n^{2}\right)$.

\section{CONCLUSION}

It is similar that three methods of rough set, D-S evidence theory and extenics describe uncertain sets. So, it has been feasible to introduce the extenics theory into information fusion. The extenics fusion can combine the matter-element analysis theory, principles of the extenics correlation functions and the Dumpster rule of combination, which can solve problems of evidence conflicts of information fusion based on D-S evidential theory and obtain of BPA functions. It can also reduce the influence of human intervention. The calculation method is simple and intuitive and is suitable for solving various kinds of evaluations.

\section{References}

[1] Wu Xiaoping, Ye Qing, Liu Lingyan. "Dempster -Shafer Theory of Evidence Based on Improved BP and Its Application". Journal of Wuhan University of Technology. vol. 29, PP. 158-161, May 2007.(in Chinese)

[2] Zhong Lu-hong, Wu Wen, Li Xingguo,et al. "Research on Fuzzy Infomation Fusion of Multi-sensor Detecting Systems". Journa 1 of Detection \& Control. vol.2, PP. 42-45, February 2003. (in Chinese)

[3] Salzenstein F, Boudraa A O. "Unsupervised multisensor data fusion approach[C], International Symposium on Signal Processing and its Applications", Kuala Lumpur, Malaysia, pp.152-154. August 2001.

[4] Zhang Qi, Gu Weikang Liu Jilin. "Vision Informayion Fusion Based on Dempster-Shafer Evidence Inference Theory in ALV". Chinese J Computers. vol. 22. pp. 193-198. February 1999 (in Chinese)

[5] Sun Quan, Ye Xiuqing, Gu Weikang. "A New Combination Rule s of Evidence Theory”. ACTA ELECTRONICA SINICA. Vol. 28. pp. 117-120. August 2000 
[6] Cai Wen, Yang Chunyan, Lin Weichu. "Extenics Engeering Methods”. Bei Jing:Science Press. 2000. (in Chinese)

[7] Lawrence A. Klein. “Theory on Fuzzy Information Fusion of Multi- sensor and Its Application”. Bei Jing: Beijing Institute of Technology Press.2004 (in Chinese)

[8] Lin Z G, Xu L Z, Huang F C, et al. "Multi-Source Monitoring Data Fusion and Assessment Model on Water Environment". Proceedings of the $3^{\text {rd }}$ International Conference on Machine Learning and Cybernetics, Shanghai, pp. 26-29. August 2004

[9] The People's Republic of China National Standards: Surface Water Quality Standards(GB3838-2002) (in Chinese) 